\title{
The microbiome: current and future view of an ancient paradigm
}

\author{
Andrew S Neish², Roberto Pacifici', Jennifer G Mulle, Colleen S Kraft ${ }^{1}$ \\ \& David S Stephens*,1
}

First draft submitted: 18 April 2017; Accepted for publication: 8 May 2017; Published online: 29 June 2017

Few areas of modern biomedical research have captured the attention and collective resources of the scientific community, as well as the imagination of the public at large, as the microbiota - the numerically vast and taxonomically complex bacterial (and viral/fungal) communities that can affect many aspects of normal biology, and by inference, health and disease. Our traditional views of the host-microbial dyad, based on an entrenched conceptualization of pathogen attack and host defense, have held sway since the time of Pasteur. The counter notion that an appropriate microbiota influences our biology in a mutually beneficial symbiotic fashion has also been considered and commented on for decades. Plant and invertebrate biologists have long studied and are familiar with this vital and necessary symbiosis between microbiota and eukaryotes, which is often the norm rather than the exception. Some vivid examples, such as the ability of symbiotic rhizobia to fix nitrogen in the root nodules of leguminous plants, or the cellulolytic activity of specialized microbes in guts of diverse herbivores, are fundamental to sustainable agriculture and indeed, ecosystems as a whole. Mammalian biologists have utilized germfree mice - maintained in physical isolator units supplied with filtered air and sterile food and water, such that the gut (and all tissues) remains devoid of a commensal microbiota for the duration of the animal's life - to demonstrate these animals exhibit an array of discernible abnormalities in a wide spectrum of physiological activities including reproductive, developmental and immune processes. Three relatively recent technical advances have led to an astonishing increase in the tangible and reliable accounting of human microbial diversity. These include experimental adaptation of PCR-based strategies to assay an array of microbial species in a single amplification; the economical availability of highthroughput sequencing; and the development of informatic algorithms to rapidly process and interpret these data. These new experimental paradigms for assessing the microbiome have since achieved appropriate scientific rigor to allow the idea of a beneficial microbiota to be fully tested. As a result, indigenous microbiota are now accepted as a discrete and inseparable component of metazoan developmental and homeostatic programs, with the presumption that during long-standing interactions, mutual co-evolution of host and microbe allowed integration of their respective signaling and gene regulatory

\section{KEYWORDS}

- host-pathogen $\bullet$ human health

- microbial communities • microbiome

- microbiota

“...indigenous microbiota are now accepted as a discrete and inseparable component of metazoan developmental and homeostatic programs..." 


\author{
“... the necessity of \\ understanding the \\ ecological origin \\ and consequences \\ of disordered \\ microbial community \\ structure (dysbiosis)."
}

circuitry into aspects of normal organismal physiology, nutrition and immune functions. The 'hygiene hypothesis' posits that the shudderingly high incidence of inflammatory and autoimmune disease (e.g., inflammatory bowel disease, asthma, juvenile onset diabetes and multiple sclerosis) and potentially metabolic disorders (adult onset diabetes, nonalcoholic steatohepatitis and obesity) may be at least partially a consequence of inadequate early life exposure to, or pharmacologic suppression of, the normal internal host-microbial ecosystem that was once an integral component of our own ancestral condition. Correlative associations of altered microbiota community structures are now linked to an astoundingly diverse range of human pathologies, not just immunological or metabolic disorders, but also neoplastic, developmental and neuropsychiatric conditions.

\section{Recent findings \& new directions in the field}

Understandably, substantial body of current microbiome science is focused on pathologies of the immune system affecting the intestine such as inflammatory bowel disease. Provocative recent work has been instrumental in characterizing specific members of the microbiota that may have direct immunoregulatory function. For example, the segmented filamentous bacteria have a particularly potent ability to induce Th17 T-cell subsets in mice [1]. Identification of such associations suggests the tantalizing possibility of exploiting specific bacteria to rationally modulate adaptive immune regulatory networks. Additionally, in innate immunity, specific taxa such as the lactobacilli possess a unique and highly conserved ability to activate epithelial movement and proliferation [2]. Furthermore, in regions of mucosal tissue injury, such proproliferative taxa, including the lactobacilli and Akkermansia, expand in the local microenvironmental conditions. The trophic effect of lactobacilli on epithelial cells [2,3] and somatic growth [4] suggests this taxa has a co-evolved multiple synergistic roles with metazoans, with biological effects that could be exploited for therapeutic purposes.

\section{- Reproductive biology}

Interestingly, lactobacilli are predominant members of the mammalian female genitourinary tract and are the first bacteria to colonize neonates. This taxa appears to confer health benefits to both. Reduced prevalence of Lactobacillus and dominance of non-Lactobacillus species is associated with pathology such as bacterial vaginosis $(\mathrm{BV})$, yeast infection and urinary tract infection. A recent landmark microbiome study using $16 S$ or metagenomic data to characterize the vaginal microbiome present in 396 multiethnic women of reproductive age [5], found the vaginal microbiota clustered into community state types (CSTs), and approximately $25 \%$ of the samples were in CST IV, where Lactobacillus was not the dominant taxon. CST IV was also associated with less acidic $\mathrm{pH}$ and higher Nugent scores (consistent with BV), although study participants were asymptomatic at enrollment. Furthermore, CST IV was overrepresented among black and Hispanic women (40.4 and $38.1 \%$, respectively). In light of prior data on weak but significant association between BV and preterm birth [6], and the long standing observation that African-American women are at higher risk for adverse pregnancy outcomes including preterm birth [7], investigation of the microbiome and CST IV in particular may offer a path toward refined associations and molecular mechanisms associated with preterm birth. Such intriguing data underscore the necessity of understanding the ecological origin and consequences of disordered microbial community structure (dysbiosis).

\section{- Bone health/osteoporosis}

The commensal microbes can also influence somatic organs distant from mucosal surfaces including the liver, lungs, joints and as recently described, the skeleton. The intestinal microbiota interacts with the somatotropic hormone axis to drive systemic skeletal growth [4]. Accordingly, animals raised in germ-free conditions have an abnormal bone mass $[4,8,9]$. Underscoring the potential role of the microbiota in the onset of osteoporosis, germ-free mice are now known to be protected against the bone loss induced by sex steroid deficiency [9]. Importantly, children with severe malnutrition possess an immature gut microbiota. Colonization of germ-free mice with such immature microbiota results in suboptimal body growth and shorter bones, whereas germfree mice on the same diet given 'mature' microbiomes of healthy children underwent normal body growth [10]. These findings point to the importance of the microbiota for growth and somatic development and suggest the utility of microbiota manipulations to address these conditions. 


\section{- Disorders of the CNS}

Other intriguing observations have implicated a role of the microbiota in neurologic and behavioral disorders and stimulated the conceptualization of a 'gut-brain axis'. Abnormalities in gut microbiota community structure have been associated with autism spectrum disorder, Parkinson's disease (PD) and other neurodegenerative and psychiatric disorders [11]. Experientially, transgenic models of PD showed markedly suppressed neuropathology when these models were conducted under germ-free conditions [12]. Mechanistically, microbial products (lipopolysaccharide or short chain fatty acids) with pro-inflammatory properties may induce CNS microglial cells with ultimate neurotoxic effects, and bacteria that affect epithelial barrier function, such as the lactobacilli, may modulate the systematic access of these potential toxic or pro-inflammatory microbial products. Alternatively, chronic redox or inflammatory stress in the gut may play a role in abnormal protein folding in the neurons of the enteric nervous system, permitting retrograde translocation to the CNS and subsequent pathology [13]. This concept is based on the presence of the characteristic neuropathological lesions of PD, Lewy bodies, recognized in the enteric nervous system, as well as functional disorders (constipation), years before the onset of CNS symptomatology.

\section{- Antibiotic \& other microbiome perturbations}

Individuals who undergo antibiotic treatment usually have a perturbation of their intestinal microbiota, which can last for months after antibiotic treatment [14]. This resultant dysbiosis creates the opportunity for bacteria that at one point may have functioned as a commensal to become dominant and create clinical disease - a pathobiont [15]. The best example of this phenomenon is Clostridium difficile infection where the individual must usually be exposed to antibiotics in addition to have acquired or have prior colonization with $C$. difficile spores. The presence of dysbiosis relates to the lack of operational taxonomic diversity in the intestine, while transfer of a healthy microbiota diversifies the microbiota leading to the recipient's recovery [16].

\section{The future needs \& horizons in the field}

The voluminous and increasing array of association studies between the microbiome and human disease must be accompanied by an equally concerted effort to understand the underlying molecular mechanisms, if this work is to achieve full translational potential.

\section{Microbiota-based interventions}

Probiotics are defined as viable microorganisms that appear to confer a health benefit when administered in adequate quantities. Probiotics are thought to alter the composition and/or the metabolic activity of the gut microbiota, regulating the immune responses in the host and enhancing epithelial barrier function. Prebiotics are nondigestible fermentable food ingredients that promote the growth of beneficial microbes and/or promote beneficial changes in the activity of the microbiome, often by providing a fermentative substrate. Wide health claims are made for both, but more work defining specific molecular mechanisms is needed. Using the example of bone health, modification (or restitution) of the gut microbiota via nutritional supplementation with prebiotics and probiotics appear to be a new promising therapeutic strategy for osteoporosis $[9,17,18]$. In theory, identification of taxa with specific cell biological or immunomodulatory activities could be candidates for potential probiotic supplementation to address multiple conditions.

At the other end of the spectrum, fecal microbiota transplant (FMT), defined as the transfer of feces from a healthy donor to a recipient, has shown to be very effective [19] in the treatment of $C$. difficile infection. In a recent randomized, placebo-controlled clinical trial, individuals with ulcerative colitis who received FMT were threetimes more likely to have a steroid-free clinical remission with endoscopic remission or response than those assigned to placebo, but a low absolute number of individuals (27\% of intervention) responded to the FMT [20]. Additionally, there have been initial anecdotal results describing the use of FMT for the elimination of multidrugresistant organisms, which colonize the intestinal microbiota. This has been followed by a clinical study in Poland where intraduodenal FMT was given to patients with blood disorders to decolonize patients [21]. In total, $75 \%$ of these patients demonstrated clearance of the multidrug-resistant organism, but this was not placebo controlled and further studies will be needed.

\section{Diagnostics}

One major limitation of schemes to modify the microbiota is that there are no current diagnostics
"In theory, identification

of taxa with specific cell biological or immunomodulatory activities could be candidates for potential probiotic supplementation to address multiple conditions." 
used clinically in real time to determine the presence of dysbiosis. While it is clear that individuals colonized by multidrug-resistant bacteria are likely to have decreased microbiota diversity [22], allowing organisms that are not typical to dominate the intestinal microbiota, little is known about how this occurs. Research is needed to define the characteristics of an 'ideal' microbiota to be able to identify what should be targeted in a diagnostic test, and ultimately, whether an intervention should occur in order to restore the microbiota. The concept of a 'microbiome disruption index' has been articulated to guide clinicians away from antibiotics that may markedly disrupt the intestinal microbiome [22], in the same way that cost is used to direct clinicians to provide equally effective but less expensive antibiotics. Overall, definitions of the parameters for normal microbial community structures in patients with various clinical syndromes will be critical to treatment and prevention of disorders associated with alteration of the microbiota. While much still needs to be done, we are in the midst of a revolution in our understanding of the role the microbiota plays in human biology and pathology.

\section{Financial \& competing interests disclosure}

The authors have no relevant affiliations or financial involvement with any organization or entity with a financial interest in or financial conflict with the subject matter or materials discussed in the manuscript. This includes employment, consultancies, honoraria, stock ownership or options, expert testimony, grants or patents received or pending, or royalties.

No writing assistance was utilized in the production of this manuscript.

\section{References}

1 Sano T, Huang W, Hall JA et al. An IL-23R/

IL-22 circuit regulates epithelial serum amyloid A to promote local effector Th17 responses. Cell 163(2), 381-393 (2015).

2 Jones RM, Luo L, Ardita CS et al. Symbiotic lactobacilli stimulate gut epithelial proliferation via Nox-mediated generation of reactive oxygen species. EMBO J. 32(23), 3017-3028 (2013).

3 Alam A, Leoni G, Quiros M et al. The microenvironment of injured murine gut elicits a local pro-restitutive microbiota. Nat. Microbiol. 1, 15021 (2016).

4 Schwarzer M, Makki K, Storelli G et al. Lactobacillus plantarum strain maintains growth of infant mice during chronic undernutrition. Science 351(6275), 854-857 (2016).

5 Ravel J, Gajer P, Abdo Z et al. Vaginal microbiome of reproductive-age women. Proc. Natl Acad. Sci. USA 108(Suppl. 1), 4680-4687 (2011).

6 Goldenberg RL, Iams JD, Mercer BM et al. The preterm prediction study: the value of new vs standard risk factors in predicting early and all spontaneous preterm births. NICHD MFMU Network. Am. J. Public Health 88(2), 233-238 (1998).

7 Kessel SS, Kleinman JC, Koontz AM, Hogue CJ, Berendes HW. Racial differences in pregnancy outcomes. Clin. Perinatol. 15(4), 745-754 (1988).

8 Sjogren K, Engdahl C, Henning P et al. The gut microbiota regulates bone mass in mice. J. Bone Miner. Res. 27(6), 1357-1367 (2012).
9 Li JY, Chassaing B, Tyagi AM et al. Sex steroid deficiency-associated bone loss is microbiota dependent and prevented by probiotics. J. Clin. Invest. 126(6), 2049-2063 (2016).

10 Blanton LV, Charbonneau MR, Salih T et al. Gut bacteria that prevent growth impairments transmitted by microbiota from malnourished children. Science 351(6275), pii:aad3311 (2016) (Epub ahead of print).

11 Sharon G, Sampson TR, Geschwind DH, Mazmanian SK. The central nervous system and the gut microbiome. Cell 167(4), 915-932 (2016).

12 Sampson TR, Debelius JW, Thron T et al. Gut microbiota regulate motor deficits and neuroinflammation in a model of Parkinson's disease. Cell 167(6), 1469-1480 (2016).

13 Braak H, de Vos RA, Bohl J, Del Tredici K. Gastric alpha-synuclein immunoreactive inclusions in Meissner's and Auerbach's plexuses in cases staged for Parkinson's disease-related brain pathology. Neurosci. Lett. 396(1), 67-72 (2006).

14 Dethlefsen L, Relman DA. Incomplete recovery and individualized responses of the human distal gut microbiota to repeated antibiotic perturbation. Proc. Natl Acad. Sci. USA 108(Suppl. 1), 4554-4561 (2011).

15 Lawley TD, Clare S, Walker AW et al. Targeted restoration of the intestinal microbiota with a simple, defined bacteriotherapy resolves relapsing Clostridium difficile disease in mice. PLoS Pathog. 8(10), e1002995 (2012).
16 Tauxe WM, Haydek JP, Rebolledo PA et al. Fecal microbiota transplant for Clostridium difficile infection in older adults. Therap. $A d v$. Gastroenterol. 9(3), 273-281 (2016).

17 Abrams SA, Griffin IJ, Hawthorne KM et al. A combination of prebiotic short- and long-chain inulin-type fructans enhances calcium absorption and bone mineralization in young adolescents. Am. J. Clin. Nutr. 82(2), 471-476 (2005).

18 Hernandez CJ, Guss JD, Luna M, Goldring SR. Links between the microbiome and bone. J. Bone Miner. Res. 31(9), 1638-1646 (2016).

19 van Nood E, Vrieze A, Nieuwdorp M et al. Duodenal infusion of donor feces for recurrent Clostridium difficile. N. Engl. J. Med. 368(5), 407-415 (2013).

20 Paramsothy S, Kamm MA, Kaakoush NO et al. Multidonor intensive faecal microbiota transplantation for active ulcerative colitis: a randomised placebo-controlled trial. Lancet 389 (10075), 1218-1228 (2017).

21 Bilinski J, Grzesiowski P, Sorensen N et al. Fecal microbiota transplantation in patients with blood disorders inhibits gut colonization with antibiotic-resistant bacteria: results of a prospective, single-center study. Clin. Infect. Dis. doi:10.1093/cid/cix252 (2017) (Epub ahead of print).

22 Halpin AL, de Man TJ, Kraft CS et al. Intestinal microbiome disruption in patients in a long-term acute care hospital: a case for development of microbiome disruption indices to improve infection prevention. $\mathrm{Am}$. J. Infect. Control 44(7), 830-836 (2016). 